\title{
Transport coefficients of dissipative particle dynamics with finite time step
}

\author{
Hiroshi Noguchi ${ }^{(a)}$ and Gerhard Gompper \\ Institut für Festkörperforschung, Forschungszentrum Jülich, 52425 Jülich, Germany
}

PACS 66.20.+d - Viscosity of liquids; diffusive momentum transport

PACS 02.70.-c-Computational techniques; simulations

PACS 47.11.-j - Computational methods in fluid dynamics

\begin{abstract}
The viscosity and self-diffusion constant of a mesoscale hydrodynamic method, dissipative particle dynamics (DPD), are investigated. The viscosity of DPD with finite time step, including the Lowe-Anderson thermostat, is derived analytically for the ideal-gas equation of state and phenomenologically for systems with soft repulsive potentials. The results agree well with numerical data. The scaling of the local relative velocity in molecular dynamics simulations is shown to be useful to obtain faster diffusion than for the DPD thermostat.
\end{abstract}

Introduction. - Soft matter systems such as polymer solutions, colloidal suspensions, vesicles, cells, and microemulsions exhibit many interesting dynamical be' haviors, where hydrodynamic flow plays an important role, as do thermal fluctuations. The characteristic length ' $(n \mathrm{~m}$ to $\mu \mathrm{m})$ and time ( $n \mathrm{~s}$ to s) scales of soft-matter systems are typically much larger than the atomistic scales. Coarse-grained molecular models and simulation methods are therefore necessary to simulate mesoscale phenomena with reasonable computational effort. Dissipative particle dynamics (DPD) [1-14] has been developed for this purpose, and has been applied to various systems such as colloids [6] polymers $[2,7,8]$ and lipid membranes [9]. DPD is ' an off-lattice hydrodynamic method, which has two main ' features: soft-repulsive interaction potentials and the pairwise version of a Langevin thermostat. Since there is no impenetrable exclude volumes, a DPD particle describes not a solvent molecule but a fluid element, which represents clusters of solvent molecules. The main motivation for the use of soft potentials is that they allows large time steps for the time evolution; however, it has been shown that the simulations have to be checked carefully in each case by monitoring the configurational temperature [15], in order to avoid artifacts due to too large time steps [5]. DPD shares many properties with direct simulation Monte Carlo [16] and multi-particle collision (MPC) dynamics [17-21] as pointed out very recently in Ref. [22].

The transport coefficients of DPD have been studied

(a) E-mail:hi.noguchi@fz-juelich.de for about a decade $[1,10-14]$. However, the viscosity of DPD has been derived analytically only for the ideal-gas equation of state in the small time-step limit [10], and partially for the Anderson-thermostat [23] version proposed by Lowe [24]. Recently, numerical integrators for the DPD thermostat $[3,4]$, which have no time-step dependence on thermodynamic properties, were proposed; they include the Lowe-Anderson thermostat (Lowe-AT) as a specific limit. However, the transport coefficients do depend on the time step. Therefore, a detailed understanding of the transport coefficients for finite time step is very important to control and tune the hydrodynamic properties of DPD fluids.

In this letter, we calculate the time-step dependence of the viscosity, and in particular investigate the contribution due to the interaction potential. The viscosity of DPD consists of three contributions, $\eta=\eta_{\text {kin }}+\eta_{\text {col }}+\eta_{\text {pot }}$. The kinetic viscosity $\eta_{\text {kin }}$, collision viscosity $\eta_{\text {col }}$, and potential viscosity $\eta_{\text {pot }}$ result from the momentum transfer due to particle displacements, collisions generated the DPD thermostat (arising from frictional interactions and thermal noise), and potential interactions, respectively. We determine these three contributions both analytically and numerically. In previous DPD simulations with a repulsive potential, the contributions of the potential interactions were often neglected in the discussion of transport coefficients. However, the potential contributes to the viscosity as well as the DPD thermostat in typical simulation conditions. We also study the self-diffusion constant 
$D$ of a DPD particle and the ratio of momentum to mass transport, which is characterized by the Schmidt number $S c=\nu / D$, where $\nu=\eta / \rho$ is the kinematic viscosity. Finally, we show that faster relaxation and larger diffusion constants $D$ can be obtained in Molecular Dynamics (MD) simulations by the rescaling of the local relative velocity to control temperature instead of a DPD thermostat.

Methods. - The DPD thermostat is a modified Langevin thermostat, where the friction and noise terms are applied to the relative velocities of the neighbor pairs. The equation of motion for the $i$-th particle with mass $m$ is given by

$$
\begin{aligned}
m \frac{d \mathbf{v}_{i}}{d t} & =-\frac{\partial U}{\partial \mathbf{r}_{i}}+f_{\mathrm{DT}} \\
f_{\mathrm{DT}} & =\sum_{j \neq i}\left\{-w\left(r_{i j}\right) \mathbf{v}_{i j} \cdot \hat{\mathbf{r}}_{i j}+\sqrt{w\left(r_{i j}\right)} \xi_{i j}(t)\right\} \hat{\mathbf{r}}_{i j}
\end{aligned}
$$

where $\mathbf{v}_{i j}=\mathbf{v}_{i}-\mathbf{v}_{j}, \mathbf{r}_{i j}=\mathbf{r}_{i}-\mathbf{r}_{j}, r_{i j}=\left|\mathbf{r}_{i j}\right|$, and $\hat{\mathbf{r}}_{i j}=\mathbf{r}_{i j} / r_{i j}$. The Gaussian white noise $\xi_{i j}(t)$ obeys the fluctuation-dissipation theorem, with average $\left\langle\xi_{i j}(t)\right\rangle=0$ and variance $\left\langle\xi_{i j}(t) \xi_{i^{\prime} j^{\prime}}\left(t^{\prime}\right)\right\rangle=2 k_{\mathrm{B}} T\left(\delta_{i i^{\prime}} \delta_{j j^{\prime}}+\delta_{i j^{\prime}} \delta_{i j^{\prime}}\right) \delta(t-$ $\left.t^{\prime}\right)$, where $k_{\mathrm{B}} T$ is the thermal energy. This thermostat is applied only in the direction $\hat{\mathbf{r}}_{i j}$ to conserve the local angular momentum. In DPD, a linear weight function $w\left(r_{i j}\right)=w_{1}\left(r_{i j}\right)$, with

$$
w_{1}\left(r_{i j}\right)=\gamma\left(1-\frac{r_{i j}}{r_{\text {cut }}}\right),
$$

is typically employed, which vanishes beyond the cutoff at $r_{i j}=r_{\text {cut }}$. Furthermore, DPD is usually combined with a soft repulsive potential [2],

$$
U=\frac{a k_{\mathrm{B}} T}{2} \sum_{i<j}\left(1-\frac{r_{i j}}{r_{\mathrm{cut}}}\right)^{2}
$$

with the same cutoff $r_{\text {cut }}$, but other potentials are also available.

The DPD equation (11) is discretized by the Shardlow's S1 splitting algorithm [3], where each thermostat of the $i j$ pair is separately integrated,

$$
\begin{aligned}
& \mathbf{v}_{i}^{\text {new }}=\mathbf{v}_{i}+\left\{-A\left(r_{i j}\right) \mathbf{v}_{i j} \cdot \hat{\mathbf{r}}_{i j}+B\left(r_{i j}\right) \xi_{i j, n}\right\} \hat{\mathbf{r}}_{i j} \\
& \mathbf{v}_{j}^{\text {new }}=\mathbf{v}_{j}-\left\{-A\left(r_{i j}\right) \mathbf{v}_{i j} \cdot \hat{\mathbf{r}}_{i j}+B\left(r_{i j}\right) \xi_{i j, n}\right\} \hat{\mathbf{r}}_{i j}
\end{aligned}
$$

with

$$
A\left(r_{i j}\right)=\frac{w\left(r_{i j}\right) \Delta t / m}{1+w\left(r_{i j}\right) \Delta t / m}, B\left(r_{i j}\right)=\frac{\sqrt{w\left(r_{i j}\right) \Delta t} / m}{1+w\left(r_{i j}\right) \Delta t / m} .
$$

The discretized Gaussian noise $\xi_{i j, n}$ is determined by $\left\langle\xi_{i j, n} \xi_{i j^{\prime} j^{\prime}, n^{\prime}}\right\rangle=2 k_{\mathrm{B}} T\left(\delta_{i i^{\prime}} \delta_{j j^{\prime}}+\delta_{i j^{\prime}} \delta_{i j^{\prime}}\right) \delta_{n n^{\prime}}$. This splitting algorithm belongs to the generalized Lowe-AT [4], because the factors $A\left(r_{i j}\right)$ and $B\left(r_{i j}\right)$ satisfy the relation $B=\sqrt{A(1-A) / m}[22]$. Thus, for $U=0$ this algorithm yields the flat radial distribution function of an ideal gas for any time step $\Delta t$, without any deviation of the kinetic temperature from the thermostat temperature. In the Lowe-AT [24], the relative velocity $\mathbf{v}_{i j} \cdot \hat{\mathbf{r}}_{i j}$ of a neighbor pair $i j$ with $r_{i j} / r_{\text {cut }}<1$ is updated by assigning a random number drawn from the Maxwell-Boltzmann distribution with the probability $\Gamma^{\prime}$ at each time step $\Delta t$ (i.e. velocities are updated with the rate $\left.\Gamma=\Gamma^{\prime} / \Delta t\right)$. When a piecewise constant weight function $w\left(r_{i j}\right)=w_{0}\left(r_{i j}\right)$, where

$$
w_{0}\left(r_{i j}\right)= \begin{cases}\gamma & \text { for } r_{i j}<r_{\text {cut }} \\ 0 & \text { otherwise }\end{cases}
$$

is employed, Eq. (4) with $\gamma \Delta t / m=1$ gives the Lowe-AT for $\Gamma^{\prime}=1$.

The viscosities are calculated from simulations of simple shear flow in three dimensions with Lees-Edwards boundary conditions [25]. We use the weight $w_{1}\left(r_{i j}\right)$, defined in Eq. (2), and the splitting algorithm (4) for the DPD simulations. However, the derived analytical expressions can be applied to other weights $w\left(r_{i j}\right)$ and other generalized Lowe-AT algorithms such as $A\left(r_{i j}\right)$ in table I of Ref. [4]. The self-diffusion constant $D$ is calculated from the mean square displacement of a particle, $\left\langle\left\{\mathbf{r}_{i}(t)-\mathbf{r}_{i}(0)\right\}^{2}\right\rangle=2 d D t$, where $d$ is the spatial dimension.

We have performed simulations with the usual soft potential (3) in order to investigate the effect of interaction potentials. The multi-time-step algorithm $[4,26]$ is employed, with a shorter time step $\delta t$ for the force $-\partial U / \partial \mathbf{r}_{i}$, so that the configurational $[5,15]$ and thermostat temperatures differ by less than $0.5 \%$. The side lengths of the simulation box are $L_{y} \geq 40 r_{\text {cut }}, L_{x}=L_{z}=10 r_{\text {cut }}$ and $L_{x}=L_{y}=L_{z}=20 r_{\text {cut }}$ for the calculation of the viscosity and the diffusion constant, respectively. The error bars of the simulation results are estimated from three independent runs. We display our simulation results in form of dimensionless quantities, indicated by a superscript, $\gamma^{*}=\gamma \tau_{0} / m, \Delta t^{*}=\Delta t / \tau_{0}, \delta t^{*}=\delta t / \tau_{0}$, and the number density $n^{*}=n r_{\text {cut }}{ }^{d}$, which corresponds to measuring length, time, viscosity, and diffusion constant of a particle in units of $r_{\text {cut }}, \tau_{0}=r_{\text {cut }} \sqrt{m / k_{\mathrm{B}} T}, \eta_{0}=\sqrt{m k_{\mathrm{B}} T} / r_{\text {cut }}^{d-1}$, and $D_{0}=r_{\text {cut }} \sqrt{k_{\mathrm{B}} T / m}$, respectively.

Viscosity of an ideal DPD gas. - First, we derive the viscosity of an ideal gas of DPD particles, with $U=0$ and $\eta_{\text {pot }}=0$, using a kinetic-theory approach. In simple shear flow with flow velocity $\mathbf{v}=\dot{\gamma} y \mathbf{e}_{x}$, the $x y$ component of the stress tensor is given by $\sigma_{x y}=\eta \dot{\gamma}$. The viscosities $\eta_{\text {kin }}$ and $\eta_{\text {col }}$ are calculated from the stress due to the kinetic and collisional contributions, respectively.

The kinetic stress $\sigma_{x y}^{\text {kin }}$ is the momentum flux due to particles crossing a plane of constant $y$; it can be calculated by following the derivation for MPC in Ref. [19]. The stress is written as

$$
\begin{aligned}
\sigma_{x y}^{\mathrm{kin}}= & \frac{m n}{\Delta t}\left\{-\int_{-\infty}^{0} d y \int_{v_{y}>-\frac{y}{\Delta t}} d \mathbf{v} v_{x} P\left(\mathbf{v}-\dot{\gamma} y \mathbf{e}_{x}\right)\right. \\
& \left.+\int_{0}^{\infty} d y \int_{v_{y}<-\frac{y}{\Delta t}} d \mathbf{v} v_{x} P\left(\mathbf{v}-\dot{\gamma} y \mathbf{e}_{x}\right)\right\},
\end{aligned}
$$



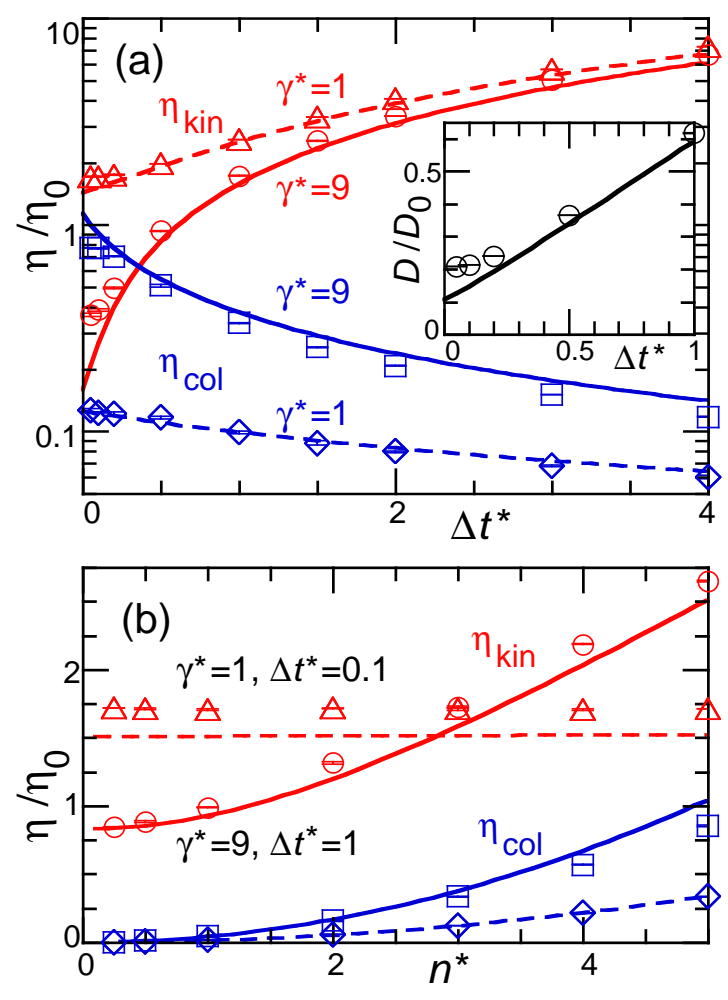

Fig. 1: (Color online) Dependence of the viscosity $\eta$ of an ideal DPD gas (with $U=0$ ) on (a) the time step $\Delta t^{*}$ at $n^{*}=3$ and (b) the number density $n$ for $\Delta t^{*}=1$. Symbols indicate simulation data for $\gamma^{*}=1(\triangle, \diamond)$ and $\gamma^{*}=9(\circ, \square)$. Lines represent the analytical results of Eqs. (8) and (12). The inset in (a) shows the dependence of the diffusion constant $D$ of the ideal DPD gas on the time step $\Delta t^{*}$ for $n^{*}=3$ and $\gamma^{*}=9$.

where $P(\mathbf{v})$ is the velocity probability distribution in the local rest frame. This stress can be rewritten as $\sigma_{x y}^{\text {kin }}=m n\left(\dot{\gamma} \Delta t\left\langle v_{x}^{2}\right\rangle / 2-\left\langle v_{x} v_{y}\right\rangle\right)$. The velocity distribution is shifted by particle streaming in the time interval $\Delta t$, so that $\left\langle v_{x} v_{y}\right\rangle \rightarrow\left\langle v_{x} v_{y}\right\rangle-\dot{\gamma} \Delta t\left\langle v_{x} v_{y}\right\rangle$. Then, the DPD collisions of Eq. (44) modify it as $\left\langle v_{x} v_{y}\right\rangle \rightarrow s\left\langle v_{x} v_{y}\right\rangle$. Thus, the self-consistency condition of a stationary shear flow is $\left\langle v_{x} v_{y}\right\rangle=s\left(\left\langle v_{x} v_{y}\right\rangle-\dot{\gamma} \Delta t\left\langle v_{x} v_{y}\right\rangle\right)$. The kinetic viscosity $\eta_{\text {kin }}$ is then given by [19]

$$
\eta_{\text {kin }}=n k_{\mathrm{B}} T \Delta t\left(\frac{1}{1-s}-\frac{1}{2}\right)
$$

The remaining task is to calculate the factor $s$ for the DPD collisions. The $i$-th particle collides with a multitude of other particles at the same time step, so that $s=\left\langle\Pi_{j} s_{i j}\right\rangle$. Eq. (4) together with a molecular chaos assumption implies $s_{i j}=1-A\left(\hat{x}_{i j}^{2}+\hat{y}_{i j}^{2}\right)+4 A^{2} \hat{x}_{i j}^{2} \hat{y}_{i j}^{2}$, where $\hat{x}_{i j}$ and $\hat{y}_{i j}$ are the components of $\hat{\mathbf{r}}_{i j}$. In an ideal gas, the local number density fluctuates around the average $n$, and the number of particles $k$ per volume $\Delta V$ is given by the Poisson distribution, $P(k)=e^{-n \Delta V}(n \Delta V)^{k} / k$ ! which implies $\left\langle c^{k}\right\rangle=\exp \{(-1+c) n \Delta V\}$ for some constant $c$. Therefore,

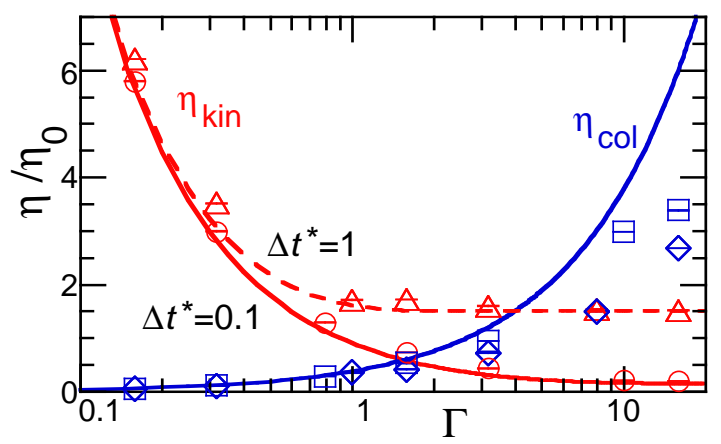

Fig. 2: (Color online) Dependence of the viscosity of an ideal DPD gas with Lowe-Anderson thermostat on the normalized collision frequency $\Gamma$. Symbols represent simulation data at $\Delta t^{*}=0.1(\circ, \square)$ and $\Delta t^{*}=1(\triangle, \diamond)$. Lines indicate the analytical results.

the factor $s$ is given by

$$
\begin{aligned}
s & =\exp \left\{n \int\left(-2 A(r) \hat{x}^{2}+4 A(r)^{2} \hat{x}^{2} \hat{y}^{2}\right) d V\right\} \\
& =\exp \left\{n \int\left(-\frac{2 A(r)}{d}+\frac{4 A(r)^{2}}{d(d+2)}\right) d V\right\} .
\end{aligned}
$$

Eqs. (8) and (9) give the kinetic viscosity $\eta_{\text {kin }}$ for a finite time step $\Delta t$. In the continuum limit $\Delta t \ll 1$, we recover the result

$$
\eta_{\text {kin }}=\frac{d m k_{\mathrm{B}} T}{2[w]_{g}}, \quad[w]_{g} \equiv \int g(r) w(r) d V
$$

of Ref. [10], where $g(r)$ is the radial distribution function, with $g(r)=1$ for the ideal gas. In the Lowe-AT, the factor $s$ is given by $s=\exp \left(-\pi b n \Gamma^{\prime}\right)$ with $b=1 / 2$ and $b=16 / 45$ in two and three spatial dimensions, respectively. In the limit $\Delta t \ll 1$ with finite $\Gamma, \eta_{\text {kin }}=k_{\mathrm{B}} T / \pi b \Gamma$.

The collisional stress $\sigma_{x y}^{\text {col }}$ is the momentum flux due to DPD collisions - determined by Eq. (4) - crossing a plane at $y=y_{0}=0$,

$$
\sigma_{x y}^{\mathrm{col}}=-n^{2} \int_{0}^{\infty} d y_{i} \int_{y_{i j}>y_{i}} d \mathbf{r}_{i j} \frac{m\left(v_{i, x}^{\text {new }}-v_{i, x}\right)}{\Delta t} .
$$

After substitution of Eq. (4) and $\left\langle v_{i j, x}\right\rangle=\dot{\gamma} y_{i j}$ into Eq. (11) and interchange of the order of integration, $\eta_{\mathrm{col}}$ is found to be

$$
\begin{aligned}
\eta_{\mathrm{col}} & =\frac{n^{2}}{2} \int d \mathbf{r} \frac{A(r) m r^{2} \hat{x}^{2} \hat{y}^{2}}{\Delta t} \\
& =\frac{n^{2}}{2 d(d+2)}\left[\frac{w r^{2}}{1+w \Delta t / m}\right]_{g} .
\end{aligned}
$$

Equation (12) gives $\eta_{\text {col }}=\left\{n^{2} / 2 d(d+2)\right\}\left[w r^{2}\right]_{g}$ in the limit $\Delta t \ll 1$. For the Lowe-AT, Eq. (12) implies $\eta_{\text {col }}=\pi m n^{2} \Gamma r_{\text {cut }}{ }^{4} / 64$ and $\eta_{\text {col }}=\pi m n^{2} \Gamma r_{\text {cut }}{ }^{5} / 75$ in two and three spatial dimensions, respectively. These results agree with the collisional viscosities obtained in Refs. [10] and [24]. 
The analytical results agree well with the numerical data, as shown in Figs. 1 and 2. As $\Delta t$ increases, $\eta_{\text {kin }}$ increases but $\eta_{\text {col }}$ decreases, just like the viscosities of MPCLangevin dynamics [22]. Although $\eta_{\text {kin }}$ is almost independent of the density $n$ at small $\Delta t, \eta_{\text {kin }}$ increases with $n$ at large $\Delta t$, see Fig. 1(b). There are small deviations between analytical and numerical results in Fig. 1. They are of the same order of magnitude as the deviations for $\Delta t \ll 1$ reported in Ref. [12], which have been explained by correlation effects between DPD collisions [12]. In the Lowe-AT, the viscosities depend on $\Delta t$ for large $\Gamma$, see Fig. 2. For $\Gamma^{\prime}=\Gamma \Delta t>1$, our theory overestimates $\eta_{\mathrm{col}}$, since the relative velocities of some $i j$ pairs are updated more than once in one time step.

Viscosity with interaction potential. - With interaction potential, an additional momentum flux crossing a plane at $y=0$, is caused by the forces $f\left(r_{i j}\right)=-\partial U / \partial r_{i j}$ between $i j$ pairs with $y_{i}>0$ and $y_{j}<0$. The potential viscosity $\eta_{\text {pot }}$ is given by

$$
\begin{aligned}
\eta_{\mathrm{pot}} & =-\frac{n^{2}}{\dot{\gamma}} \int_{0}^{\infty} d y_{i} \int_{y_{i j}>y_{i}} d \mathbf{r}_{i j} g\left(r_{i j}\right) f\left(r_{i j}\right) \hat{x}_{i j} \\
& =-\frac{n^{2}}{2 \dot{\gamma}} \int d V g(r) f(r) \hat{x} y,
\end{aligned}
$$

which is the potential term of the Irving-Kirkwood formula of the viscosity [27]. The potential also modifies $\eta_{\text {kin }}$ with an additional velocity relaxation, while $\eta_{\text {col }}$ can be calculated by Eq. (12) with non-uniform $g(r)$. The viscosity with an interaction potential has been derived analytically for some cases [28], but is generally very complicated. Therefore, we employ a simple phenomenological expression instead, and focus on the explanation of qualitative dependences.

In the molecular-chaos approximation, the velocity auto-correlation function of a particle in a gas shows an exponential decay, $\langle v(t) v(0)\rangle=\exp (-\phi t)$. This behavior corresponds to the assumption of a Langevin equation; $d v / d t=-\phi v+\sqrt{\phi} \xi(t) / m$ for the particle velocity $v$ in the local rest frame. For $\Delta t \ll 1$, the DPD collisions generate an auto-correlation function with an initial exponential decay (for small times $t$ ) with $\phi_{\mathrm{DPD}}=n[w]_{g} / d m$. Here, we assume that the potential also generates an exponential auto-correlation function with rate $\phi_{\text {pot }}$, although the auto-correlation function determined numerically is not exponential, and shows larger deviation from an exponential decay for larger potential strengths $a$ or particle densities $n$. Then, the kinetic viscosity $\eta_{\text {kin }}$ is given by

$$
\eta_{\text {kin }}=\frac{n k_{\mathrm{B}} T}{2\left(\phi_{\mathrm{pot}}+\phi_{\mathrm{DPD}}\right)},
$$

compare Eq. (10).

In order to estimate $\eta_{\text {pot }}$, an expression for the correlations of $i j$ pairs is required. We mimic the potential
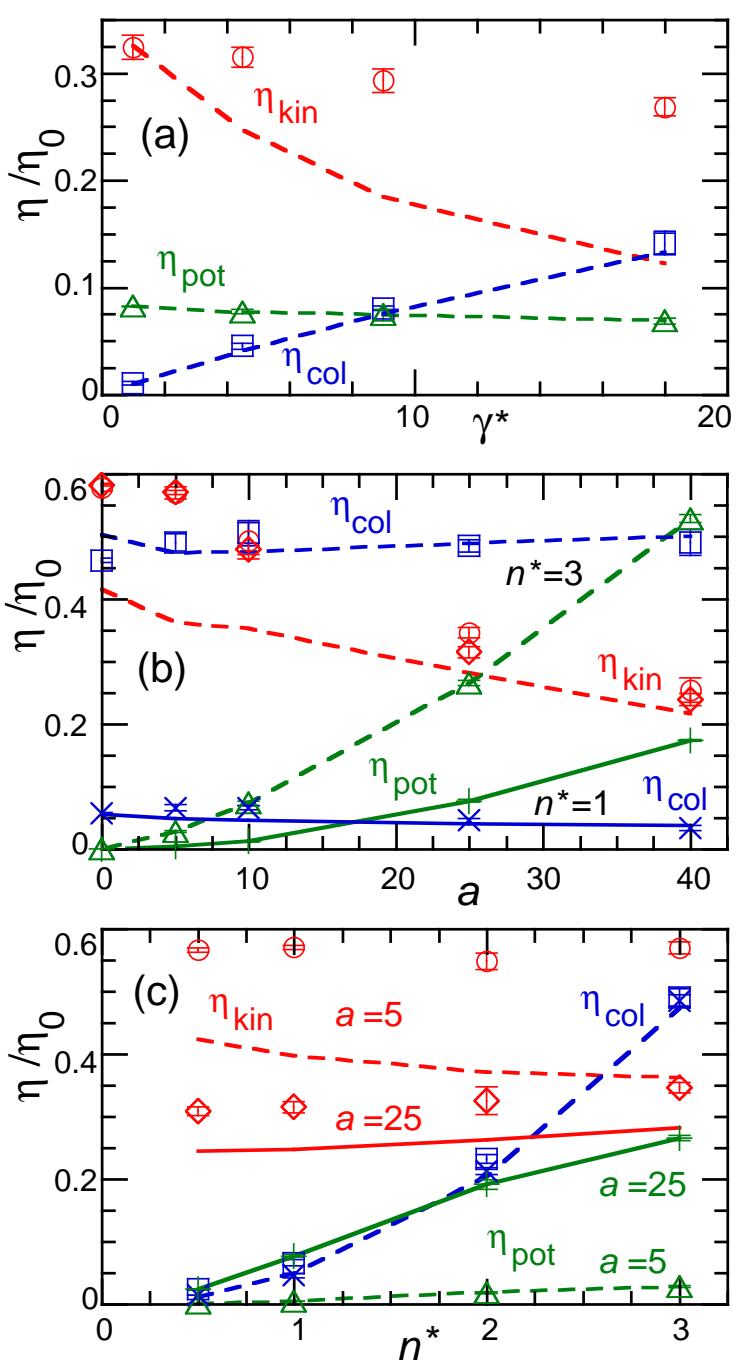

Fig. 3: (Color online) Dependence of the viscosity of DPD with soft potentials, Eq. (3), on (a) friction coefficient $\gamma^{*}$ for $n^{*}=1$ and $a=25$, (b) potential strength $a$ for $\gamma^{*}=4.5$, and (c) number density $n$ for $\gamma^{*}=4.5$. In all cases, $\delta t^{*}=0.01$, and $\Delta t^{*}=0.1$. Symbols indicate simulation data for (b) $n^{*}=3$ $(\circ, \square, \triangle)$ and $n^{*}=1(\diamond, \times,+)$, and (c) $a=5(\circ, \square, \triangle)$ and $a=25(\diamond, \times,+)$. Lines for $\eta_{\text {col }}$ represent the analytical results of Eq. (12). Lines for $\eta_{\text {kin }}$ show the results of Eq. (14) with $\phi_{\text {pot }}$ fitted by Eq. (16). Lines for $\eta_{\text {pot }}$ are guides to the eye.

contribution by a DPD thermostat,

$$
m \frac{d \mathbf{v}_{i}}{d t}=\sum_{j \neq i}\left\{-\gamma_{\text {pot }}\left|f\left(r_{i j}\right)\right| \mathbf{v}_{i j} \cdot \hat{\mathbf{r}}_{i j}+\sigma \xi_{i j}(t)\right\} \hat{\mathbf{r}}_{i j},
$$

where $\sigma=\sqrt{\gamma_{\text {pot }}\left|f\left(r_{i j}\right)\right|}$, since the restoring force should be proportional to $\left|f\left(r_{i j}\right)\right|$, and $v_{i j}$ in the direction $\hat{\mathbf{r}}_{i j}$. Following the derivation of Eq. (12) with $\phi_{\text {pot }}=$ $n \gamma_{\text {pot }}[|f|]_{g} / d m$, we obtain the viscosity

$$
\eta_{\mathrm{pot}}=\frac{n m \phi_{\mathrm{pot}}\left[|f| r^{2}\right]_{g}}{2(d+2)[|f|]_{g}}
$$

Thus, the viscosities $\eta_{\text {kin }}$ and $\eta_{\text {pot }}$ can be estimated by 
Eqs. (14) and (16) with the parameter $\phi_{\text {pot }}$ and the radial distribution function $g(r)$.

Figure 3 shows the viscosities of the DPD fluid with interaction potential. We calculate $g(r)$ from equilibrium simulations, fit $\phi_{\text {pot }}$ to $\eta_{\text {pot }}$, and then estimate $\eta_{\text {kin }}$ from Eq. (14). This underestimates $\eta_{\text {kin }}$, but reproduces very well the qualitative dependence on friction coefficient $\gamma$, potential strength $a$, and number density $n$. The kinetic viscosity $\eta_{\text {kin }}$ decreases with increasing $\gamma$ or $a$. The potential viscosity $\eta_{\text {pot }}$ is almost independent of $\gamma$ and increases with $a$. The collision viscosity $\eta_{\text {col }}$ is almost independent of $a$ and shows very good agreement between the theory and simulations.

Diffusion. - Next, we derive the self-diffusion constant $D$ of an ideal gas of DPD particles (with $U=0$ ) for finite time steps. Following the derivation of Eq. (9), we find that the velocity correlation for one step is given by $\left\langle v_{x}(t+\Delta t) v_{x}(t)\right\rangle=\exp \left(-n[A]_{g} / d\right)$. Under the molecular chaos assumption, i.e. $\left\langle v_{x}(k \Delta t) v_{x}(0)\right\rangle=\left\langle v_{x}(\Delta t) v_{x}(0)\right\rangle^{k}$, the diffusion constant is then given by

$$
D=\frac{k_{\mathrm{B}} T \Delta t}{m}\left(\frac{1}{1-\exp \left(-n[A]_{g} / d\right)}-\frac{1}{2}\right) .
$$

In the limit $\Delta t \ll 1$, the diffusion constant becomes $D=d k_{\mathrm{B}} T / n[w]_{g}$, in agreement with the result of Ref. [10]. However, the velocity auto-correlation function $\left\langle v_{x}(k \Delta t) v_{x}(0)\right\rangle$ with large dimensionless friction coefficient $\gamma^{*}$ has a long-time tail due to the hydrodynamic interactions [11], and the diffusion constant $D$ becomes larger than the value in Eq. (17). This underestimation of $D$ is seen at small $\Delta t$ in the inset of Fig. 1(a).

Since the kinetic contribution to the kinematic viscosity $\eta_{\text {kin }} / \rho$ is roughly proportional to $D$, the relation $\eta_{\text {kin }} \ll \eta_{\text {col }}+\eta_{\text {pot }}$ at large friction coefficient $\gamma^{*}$ or potential strength $a$ yields large Schmidt numbers $S c$ in DPD. On the other hand, small $\gamma^{*}$ and $a$ gives $S c<1$, e.g. $S c=\eta_{\text {kin }} / \rho D=1 / 2$ for $\Delta t \ll 1$ and $\eta_{\text {kin }} \gg \eta_{\text {col }}+\eta_{\text {pot }}$. Sufficiently large $S c$ yields hydrodynamic behavior. For example, a large Schmidt number is required in polymer simulations to produce Zimm dynamics [29] — where the relaxation time $\tau_{p}$ of a mode with mode number $p$ is expected to scale as $\tau_{p} \sim\left(N_{m} / p\right)^{3 / 2}$ — with moderate chain lengths $N_{m}$ for an ideal chain, as demonstrated in MPC simulations with $S c \simeq 10$ [20]. Zimm dynamics was also reported from DPD simulations with the most typical parameters $n^{*}=3, a=25$ and $\gamma^{*}=4.5$ [8] or 5.6 [7]; however, the variation of the Zimm exponent with temperature observed in Ref. [8] seems to indicate that the simulations were performed in the region between the Rouse and Zimm regimes. From our results above, we obtain $S c=1.7$ and $\eta_{\text {col }}+\eta_{\text {pot }} \simeq 2 \eta_{\text {kin }}$ at $n^{*}=3, a=25$, $\gamma^{*}=4.5$ with $\delta t=0.01$ and $\Delta t=0.1$. Thus, this parameter set is indeed in the crossover region between gas-like and liquid-like behavior.

Other Thermostats. - To simulate the hydrodynamic behavior of complex fluids, dimensionless hydro-

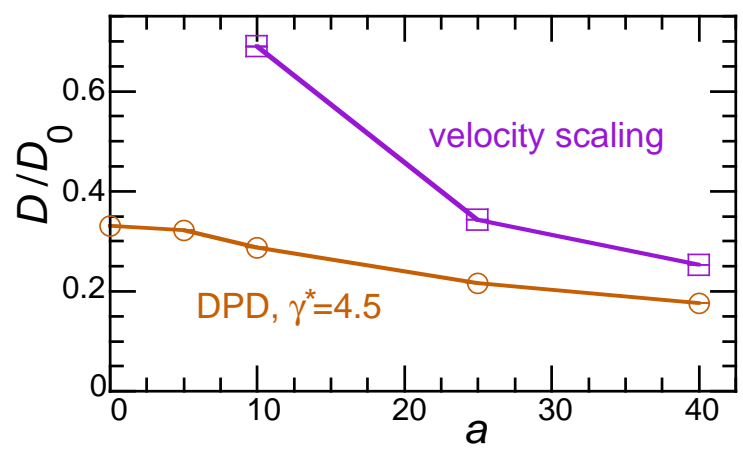

Fig. 4: (Color online) Dependence of the diffusion constant $D$ on the strength $a$ of the soft potential (3), for $n^{*}=3$, $\delta t^{*}=0.01$, and $\Delta t^{*}=0.1$. The velocity rescaling is performed with time step $\Delta t^{*}$ and cell size $l_{\mathrm{c}}=r_{\text {cut }}$. In comparison, the diffusion constant $D$ of DPD with $\gamma^{*}=4.5$ is also shown.

dynamic quantities, such as the Reynolds number and the Schmidt number $S c$, typically have to be adjusted to match experimental conditions. To study low-Reynoldsnumber flows of soft matter and biological systems, high viscosity is often required. On the other hand, DPD simulations are also often employed to study equilibrium properties. In this case, faster diffusion and lower viscosity is advantageous, since it provides faster relaxation into the equilibrium state. Recently, a Nosé-Hoover-type thermostat for the relative velocities of neighbor pairs was proposed $[30,31]$, where the momentum is locally conserved. Its main idea is to thermostat systems, but to less disturb the original hydrodynamic transport properties (in the absence of any thermostat). However, the Nosé-Hoover thermostat usually has to be combined with another thermostat to keep the temperature constant, when a system includes a potential with strong $C^{2}$ discontinuity like Eq. (3).

The scaling of velocities [25] is an easy way to control the temperature in MD simulations in thermal equilibrium. In order to retain hydrodynamic properties, e.g. under flow, the main issue is momentum conservation, i.e. how to determine the velocity of the local rest frame. We suggest to employ the velocity scaling of the MPC method [17], which can be used independent of the MPC collision procedure. The particles are sorted into the cells of a cubic lattice with lattice constant $l_{\mathrm{c}}$, and the local flow velocity is identified with the velocity $\mathbf{v}_{\mathrm{c}}^{\mathrm{G}}$ of the center of mass of all particles in a cell. Then, the relative velocities $\mathbf{u}_{i}=\mathbf{v}_{i}-\mathbf{v}_{\mathrm{c}}^{\mathrm{G}}$ are rescaled as $\mathbf{u}_{i} \rightarrow \mathbf{u}_{i} \sqrt{d\left(N-N_{\mathrm{c}}\right) k_{\mathrm{B}} T / m \sum_{i} \mathbf{u}_{i}^{2}}$, where $N$ is the total number of particles and $N_{\mathrm{c}}$ is the number of cells occupied by particles. The cells are randomly shifted before each scaling step to ensure Galilean invariance [18]. The velocity scaling gives faster diffusion than DPD as shown in Fig. 4. This is particularly important for solvents with Lennard-Jones-type interactions, where the frictional contributions of a DPD thermostat adds up with an already high viscosity in classical MD. Velocity rescaling can produce temperature gradients in flow due 
to a locally inhomogeneous energy dissipation. To reduce these gradients, a local version of rescaling procedure can be employed. To do so, many cells are grouped into larger bins, typically arranged sequentially in layers or columns, and the rescaling is performed individually for each bin. Alternatively, gradients on the cell scale can be avoided by velocity scaling with a Monte Carlo scheme [21], where the scaling factor fluctuates stochastically in each cell to reproduce the correct kinetic energy distributions.

Summary. - We have studied the viscosity of DPD with finite time step, both analytically and numerically. The analytical results agree very well with the simulation data. Our theoretical results for the viscosity can be generalized straightforwardly to other DPD methods, such as DPD with a multibody thermostat [22]. Thus, we have shown that by varying the time step $\Delta t$ and the friction coefficient $\gamma$, the dynamic properties of a DPD solvent can be tuned, while thermodynamic properties remain unaffected.

Furthermore, we have shown that the velocity rescaling method, which is routinely employed in MPC, can be adapted to MD simulations. It respects Galilean invariance and disturbs the original hydrodynamics much less than a DPD thermostat.

$$
* * *
$$

We thank M. Ripoll for helpful discussions. The partial support of this work by the DFG through the priority program "Nano- and Microfluidics" is gratefully acknowledged.

\section{REFERENCES}

[1] Hoogerbrugge P. J. and Koelman J. M. V. A., Europhys. Lett., 19 (1992) 155.

[2] Groot R. D. and Warren P. B., J. Chem. Phys., 107 (1997) 4423.

[3] Shardlow T., SiAM J. Sci. Comput., 24 (2003) 1267.

[4] Peters E. A. J. F., Europhys. Lett., 66 (2004) 311.

[5] Allen M. P., J. Phys. Chem. B, 110 (2006) 3823.

[6] Boek E. S., Coveney P. V., Lekkerkerker H. N. W. and van Der Schoot P., Phys. Rev. E, 55 (1997) 3124.

[7] Spenley N. A., Europhys. Lett., 49 (2000) 534.

[8] Jiang W., Huang J., Wang Y. and Laradji M., J. Chem. Phys., 126 (2007) 044901.

[9] Venturoli M., Sperotto M. M., Kranenburg M. and Smit B., Phys. Rep., 437 (2006) 1.

[10] Marsh C. A., Backx G. and Ernst M. H., Phys. Rev. $E, 56$ (1997) 1676.

[11] Español P. and Serrano M., Phys. Rev. E, 59 (1999) 6340.

[12] Masters A. J. and Warren P. B., Europhys. Lett., 48 (1999) 1.

[13] Ripoll M., Ernst M. H. and Español P., J. Chem. Phys., 115 (2001) 7271.

[14] Visser D. C., Hoefsloot H. C. J. and Iedema P. D., J. Comput. Phys., 214 (2006) 491.
[15] Rugh H. H., Phys. Rev. Lett., 78 (1997) 772.

[16] Bird G. A., Molecular gas dynamics (Clarendon, Oxford) 1976.

[17] Malevanets A. and Kapral R., J. Chem. Phys., 110 (1999) 8605.

[18] T. Ihle and D. M. Kroll, Phys. Rev. E, 63 (2001) 020201(R).

[19] Kikuchi N., Pooley C. M., Ryder J. F. and Yeomans J. M., J. Chem. Phys., 119 (2003) 6388.

[20] Ripoll M., Mussawisade K., Winkler R. G. and Gomprer G., Europhys. Lett., 68 (2004) 106.

[21] Hecht M., Harting J., Ihle T. and Herrmann H. J., Phys. Rev. E, 72 (2005) 011408.

[22] Noguchi H., Kikuchi N. and Gompper G., Europhys. Lett., 78 (2007) 10005.

[23] Andersen H. C., J. Chem. Phys., 72 (1980) 2384.

[24] Lowe C. P., Europhys. Lett., 47 (1999) 145.

[25] Allen M. P. and Tildesley D. J., Computer simulation of liquids (Clarendon Press, Oxford) 1987.

[26] Tuckerman M., Berne B. J. and Martyna G. J., J. Chem. Phys., 97 (1992) 1990.

[27] Irving J. and Kirkwood J., J. Chem. Phys., 18 (1950) 817.

[28] RÉsibois P. and DE LeEner M., Classical kinetic theory of fluids (Wiley, New York) 1987.

[29] Doi M. and EDwards S. F., The theory of polymer dynamics (Clarendon Press, Oxford) 1986.

[30] Groot R. D. and Stoyanov S. D., J. Chem. Phys., 122 (2005) 114112.

[31] Allen M. P. and Schmid F., arXiv:cond-mat/0606511, (2006) . 